\title{
The orbital signatures of HMXBs in optical and X-ray measurements - the BeX-SFXT link
}

\author{
Antony Bird* \\ Physics and Astronomy, Faculty of Physical and Applied Sciences, University of Southampton, \\ SO17 1BJ, UK. \\ E-mail: ajbirdesouthampton.ac.uk
}

\begin{abstract}
The prospects for long-term optical monitoring of HMXB as a means of obtaining diagnostic information on orbital and accretion processes are discussed. Of the various classes of HMXB, the Be X-ray binaries have been studied in greatest detail with optical ground-based monitoring, with good results. Optical signatures of both orbital modulation and other mechanisms such as decretion disk asymmetries and pulsations of the donor star can be detected, and various strategies are discussed to identify these contributions to the optical light curve. A few of the recently discovered SFXT class show characteristics similar to the Be-star systems, in that a semi-stable equatorial enhancement is seen in the stellar wind. In principle, these systems should be good candidates for long-term monitoring campaigns.
\end{abstract}

The Extreme and Variable High Energy Sky - extremesky2011,

September 19-23, 2011

Chia Laguna (Cagliari), Italy

\footnotetext{
* Speaker.
} 


\section{Introduction}

High mass X-ray binaries (HMXB) are stellar systems in which a compact object, normally a neutron star, accretes material that is transferred to it from a high-mass companion star. This mass transfer can take place via a number of physical mechanisms. In a few supergiant systems, Roche lobe overflow provides a direct and persistent accretion flow onto the neutron star, but in most HMXBs, mass transfer is via a dense stellar wind, and the geometry of that wind creates the various observational classes observed. Supergiant stars have a strong, quasi-isotropic and inhomogeneous wind, and a neutron star orbiting within that wind will accrete at varying rates, giving rise to an erratic, flaring X-ray light curve. In Be stars, however, the stellar wind is in the form of a semi-stable equatorial decretion disc which the orbiting neutron star encounters only once (or maybe twice) per orbit, and so the X-ray emission is in the form of periodic outbursts that vary in amplitude depending on the size of the decretion disc at the time of the encounter. The classification of HMXB is often illustrated by the use of the Corbet diagram (Figure 1), which plots the neutron star spin period and orbital period for HMXBs [1]. Although the precise mechanisms causing their placement within the Corbet diagram are still not not well understood, the three different mass transfer mechanisms place sources in distinct regions of the diagram.

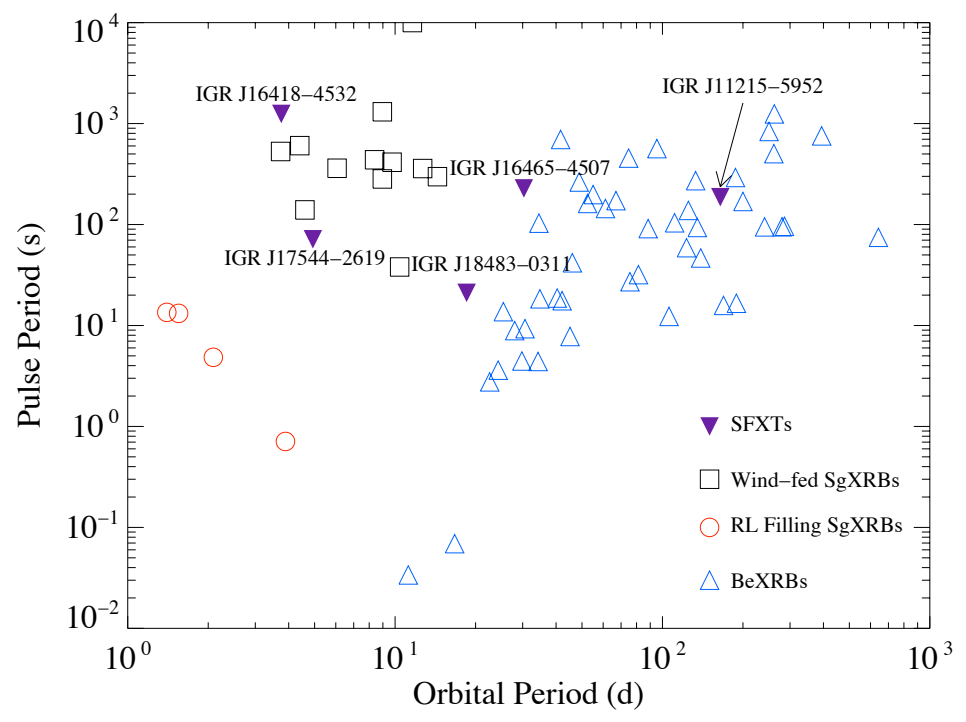

Figure 1: Corbet diagram showing the segregation of HMXB with different mass transfer mechanisms

Since the spin and orbital periods of many HMXB are directly measurable from the X-ray observations alone, this diagram provides a simple method to quickly establish the accretion mechanism in a newly discovered source. More recently, however, this relatively simple scheme has been complicated by attempts to characterise the Supergiant Fast X-ray Transient (SFXT) systems. These systems have been discovered (for example $[2,3]$ ) mostly on the basis of their X-ray characteristics: particularly high dynamic range of $\sim 100-1000$ on short timescales, encompassing fast flaring episodes lasting a few hours and deep quiescence. Follow-up observations (for example $[4,5]$ showed their HMXB nature, and long-term studies allowed some orbital and spin periods to 
be determined, showing them to be widely distributed on the Porb-Pspin diagram. It may be that the production channels for the SFXT systems are not all the same, and several subclasses exist, but one intriguing feature is the 'intermediate' systems which do not lie in any of the traditional regions of the Corbet diagram. Unfortunately, the term intermediate is itself used quite loosely in connection with SFXTs, and can also be used to denote those systems exhibiting less extreme behaviour in terms of dynamic range, but here I use the term to indicate those systems that lie in previously unoccupied regions of the Corbet diagram (see, for example, IGR J18483-0311 in Figure 1). Whatever the origin of the SFXTs, it is clear that more systems need to be characterised before a realistic study can be performed and thus the determination of orbital and spin periods for more systems is an essential step. Since the X-ray outbursts from SFXT are by no means regular, it may require the accumulation of many, many orbits of data before statistics will allow determination of the orbital period. In these cases, the optical band, which has been successfully used to determine the orbital period in other HMXB, may be an appropriate additional tool.

In this paper, I will summarise the progress made in using optical data to determine orbital periods of the Be/X-ray binary systems, and by analogy with some of the SFXT systems, indicate the role that long-term monitoring may play in future SFXT studies.

\section{Optical monitoring of Be/X-ray binaries}

The long-term optical behaviour of Be/X-ray binaries as a population of objects is quite well studied, mostly through the use of the MACHO and OGLE projects that have provided long-term monitoring of objects in the LMC and SMC. These projects have provided I-band and V-band monitoring on one-day cadence over 15 years now. The optical properties of the Be/X-ray binaries are dominated by the star itself, and the emission of the decretion disk. The flux from the disk is expected to dominate the I-band flux from the system, and this band is used prevalently to study the mass transfer within the systems. The decretion disk can also be observed in detail using $\mathrm{H} \alpha$ spectroscopy, which allows the long-term photometry data to be placed in a more complete context.

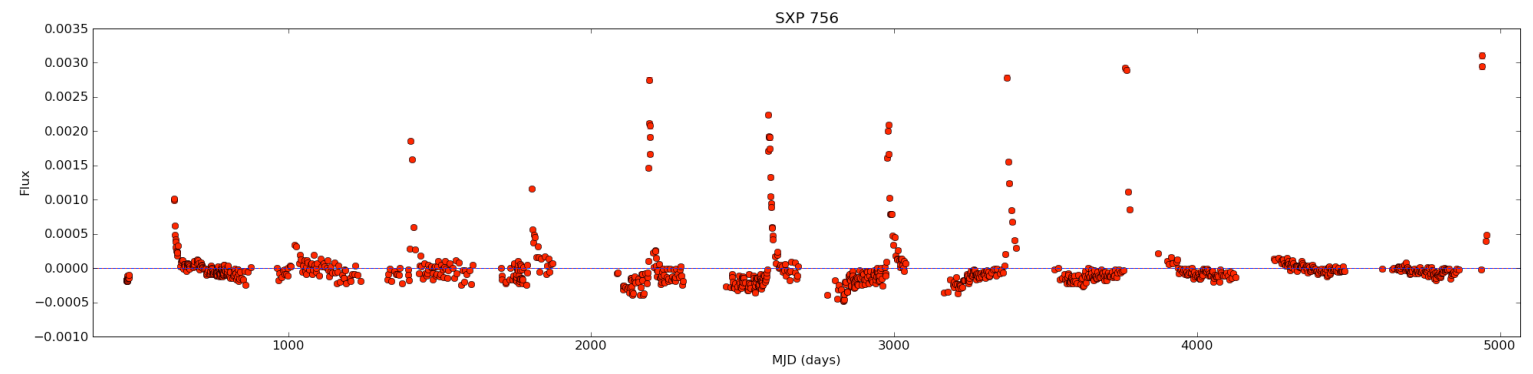

Figure 2: OGLE-II and OGLE-III I-band light curve of an SMC Be/X-ray binary

Figure 2 shows many of the key features seen in long-term monitoring. On top of slow variations, indicating the growth and decline of the decretion disk, large peaks are seen. These peaks result from the periastron passage of the neutron star in its 393.6 day orbit causing a disruption of the decretion disk, and the resulting change in disk area is seen as a rapid increase in optical flux. The response of the disk, and its recovery after the passage of the neutron star give rise to a fast 
rise and exponential decay (FRED) like profile in the light curve. Such a profile is seen in many systems, and seems to be strong indicator of an orbital modulation. Underlying the large-scale variations, and only really visible outside of the periastron flares, are other sinusoidal variations seen on a timescale of $\sim 11 \mathrm{~d}$. These have been attributed to pulsations of the Be star itself, probably at a period of $0.9 \mathrm{~d}$, which manifests itself at a period of around 11 days as a result of aliasing with the 1 day sampling of the OGLE dataset. This effect has been observed in other systems e.g. [6], and causes difficulties when using optical monitoring alone to try to determine orbital periods. In SXP756, since the orbital period is very clear (the $393.6 \mathrm{~d}$ periodicity is seen in both optical and $\mathrm{X}$-ray light curves) the $11 \mathrm{~d}$ period is unlikely to be misinterpreted. In other systems, however, a single detected period could be either orbital, or an aliased pulsation, and this degeneracy cannot be broken without either a light curve with a different sampling, or further information on the system itself.

The folded light curve shape can be a good way to break the degeneracy concerning the possible origins of periodicities in the optical light curves. As shown already for SXP756, an orbital modulation tends to be both asymmetric (following a FRED-like profile) and covers a short span in phase. The peaks in folded light curves deriving from sinusoidal variations of aliased pulsations, on the other hand, have a symmetric folded light curve and a phase span of 0.5 , as expected for a simple sinusoid. Thus inspection of the folded light curve, at least for some systems, provides a way to identify true orbital signatures in these systems. The caveat attached to this method comes about because this is probably only true of rather eccentric systems, while a more circular orbit would give a less pronounced 'impact' with the decretion disk, and a less characteristic folded light curve profile.

\section{Prospects for SFXT optical monitoring}

Since it is well established that Be/X-ray binaries can be studied by optical monitoring, are there common features of the SFXTs that could be studied in the same way? Certainly, two sources within the SFXT class show features in their X-ray light curves that have been associated with equatorially enhanced winds. IGR J11215-5952 shows evidence for a persistent equatorial enhancement in the stellar wind around the donor star [7], which is orbited every 164.6d and gives rise to regular X-ray outbursts near periastron, and a very deep quiescence away from it. Similarly, the folded light curve for IGR J17391-3021 (Figure 3) shows several features [8], interpreted as periastron passages through a relatively isotropic wind component, plus two passages through an enhanced disk-like region giving rise to the two side-peaks in the folded light curve.

By analogy with the Be-X-ray systems, these two systems could perhaps be expected to exhibit an orbitally modulated optical behaviour if the interaction with the disk-like structure is significant. However, there are many differences, all of which make the prospects for optical modulation less certain:

- the disk itself is likely to be of much lower density than a Be star decretion disk, even if the two systems described above show evidence of a stable disk, which is an absolute prerequisite for detecting modulation 


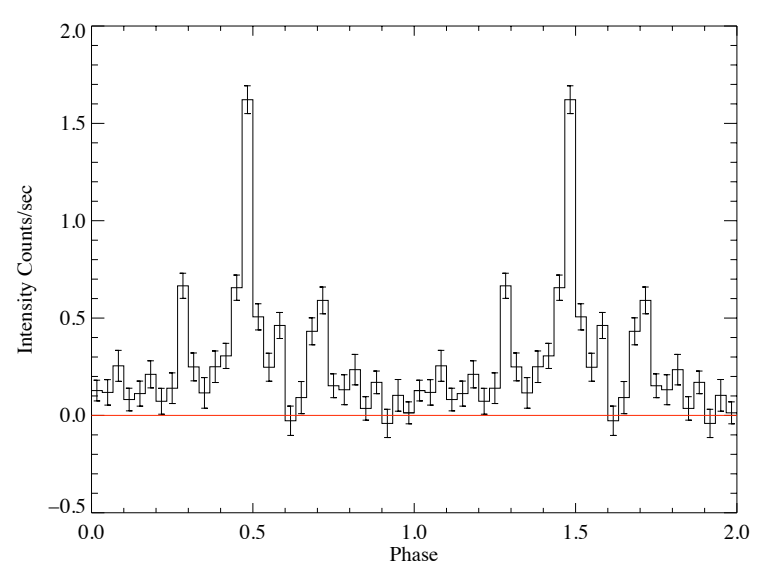

Figure 3: Folded hard X-ray light curve for XTE J1739-302

- the heating of disk from the supergiant donor will be more extreme than in a Be system, and the reference I-band used for Be/X-ray systems may be less appropriate

- The supergiant itself will be a strong emitter, and any fluctuations in the stellar flux will be likely to obscure periodic emission from the disk

Long-term optical monitoring is unlikely to be a suitable technique for all SFXTs, as evidence for equatorial disk-like winds is by no means ubiquitous in the class. In fact IGR J17391-3021 and IGR J11215-5952 are the only two systems to (so far) show such evidence. In the shorter period systems, the X-ray flaring behaviour is believed to derive from accretion of a isotropic, but inhomogeneous 'clumpy' wind, and there is no reason to expect orbital modulation of the optical flux in these systems.

Limited programmes of long-term photometric observations have been attempted using relatively small robotic telescopes. Such telescopes offer flexible operation, and occasional highcadence sampling of the optical light curves of HMXB. However, the absolute photometry accuracy is insufficient to detect the small variations likely to result from neutron star interactions with the equatorial wind in SFXT. More promising is that fact that new survey programmes such as OGLE-IV are now operating over larger areas of the Galactic plane, offering the prospect of highaccuracy monitoring spanning years. Programmes like OGLE have already shown their value for Be/X-ray studies, not only for detection of orbital parameters, but also in studying the more detailed interactions between the components of the binary systems. A similar approach for SFXTs should certainly be considered.

\section{References}

[1] R. Corbet. 1986, MNRAS, 376, 759

[2] V. Sguera, A. Bazzano, A.J. Bird, et al., 2006, MNRAS, 646, 452

[3] I. Negueruela, D.M. Smith, P. Reig, S. Chaty, J.M. Torrejón, 2005, in Proceedings of the The X-ray Universe, ESA SP-604, 165 
[4] I. Negueruela et al., 2005, Astronomers Telegram, 470.

[5] I. Negueruela, D.M. Smith, T.E. Harrison, J.M. Torrejón, 2006, ApJ, 638. 982

[6] P.C. Schmidtke, A.P. Cowley, 2006, Astronomers Telegram, 716.

[7] L. Sidoli, P. Romano, S. Mereghetti, et al., 2007, A\&A, 476, 1307

[8] S.P. Drave, D.J. Clark, A.J.Bird, et al., 2010, MNRAS, 4091220. 